\title{
Estimação de Parâmetros de Modelos Bouc-Wen via Algoritmos Evolutivos para Compensação de Histerese
}

\author{
Lucas A. Tavares* Petrus E. O. G. B. Abreu* \\ Luis A. Aguirre* \\ * Programa de Pós-Graduação em Engenharia Elétrica, Universidade \\ Federal de Minas Gerais, MG (e-mails: amarallucas@ufmg.br, \\ petrusabreu@ufmg.br,aguirre@ufmg.br)
}

\begin{abstract}
Hysteresis is a hard nonlinearity which challenges the design of control systems. In control or compensation problems, it is desirable to atenuate for this effect which can be made via models. A classical hysteretic structure is known as Bouc-Wen model. Parameter estimation based on data for this model yields a non-convex optimization problem. In this work, an evolutive approach is used to estimate parameters. Such an approach enables one to insert an additional parameter that results in modified models. With these models, a method from the literature is used to compensate hysteresis in two examples: a numerical simulation and a physical system. The obtained results show: i) both estimated models, classical and modified, provide hysteresis compensation, and ii) the modified models are systematically superior to achieve this aim.

Resumo: Histerese é uma não linearidade severa e desafiadora no contexto de controle. Em problemas de controle ou compensação, é desejável atenuar esse efeito, o que pode ser feito via modelos. Uma estrutura clássica para representar histerese é o modelo de Bouc-Wen. A estimação dos parâmetros desse modelo a partir de dados resulta em um problema não convexo. Neste trabalho, utiliza-se uma abordagem evolutiva para a estimação desses parâmetros o que permite a inserção de um parâmetro adicional, resultando em modelos modificados. De posse dos modelos, um método disponível na literatura é usado para compensar a histerese em dois exemplos: uma simulação numérica e um sistema físico. Os resultados obtidos mostram que i) os modelos estimados, tanto o clássico como o modificado, permitiram realizar a compensação da histerese, e ii) os modelos modificados mostraram-se sistematicamente superiores para esse fim.
\end{abstract}

Keywords: Parameter Estimation; Evolutionary Algorithms; Bouc-Wen Model; Compensation; Hysteresis.

Palavras-chaves: Estimação de Parâmetros; Algoritmos Evolutivos; Modelo Bouc-Wen; Compensação; Histerese.

\section{INTRODUÇÃO}

Histerese é um fenômeno severamente não linear que pode ser observado em diversos contextos: sensores e atuadores (Visone, 2008), estruturas mecânicas (Swevers et al., 2000), variáveis econômicas (Cross et al., 2009) e sistemas biológicos (Sha et al., 2003). Nesse fenômeno, a saída não depende apenas dos valores atuais do estado e da entrada, mas também do histórico da entrada. Assim, tal não linearidade é associada à existência de um laço no plano entrada $\times$ saída quando o sistema é excitado em baixas frequências (Ikhouane and Rodellar, 2007). Em controle, esse comportamento é indesejável, dado que tende a dificultar a redução de erros no contexto de rastreamento (Morris, 2011).

Bouc (1967) formulou um modelo capaz de representar sistemas histeréticos para vibrações mecânicas que foi estendido por Wen (1976). Atualmente, essa estrutura é conhecida como modelo de Bouc-Wen (BW) e é dada por:

\footnotetext{
* Este trabalho foi realizado com apoio do CNPq e da FAPEMIG.
}

$\dot{h}(t)=\alpha_{\mathrm{bw}} \dot{u}(t)-\beta_{\mathrm{bw}}|\dot{u}(t)||h(t)|^{n-1} h(t)-\gamma_{\mathrm{bw}} \dot{u}(t)|h(t)|^{n}$

sendo $h(t)$, a variável de estado da histerese, $u(t)$, a excitação mecânica e os parâmetros $\alpha_{\mathrm{bw}}, \beta_{\mathrm{bw}}, \gamma_{\mathrm{bw}}$ e $n$ determinam o laço. Alguns trabalhos consideram todos os sinais do modelo (1) como positivos, o que não afeta o resultado caso os parâmetros possam assumir qualquer sinal algébrico na etapa de estimação, como é o caso no presente trabalho. Pelo fato desse modelo ser capaz de representar uma ampla gama de laços (Ikhouane and Rodellar, 2007), $u(t)$ não precisa ser necessariamente uma excitação mecânica.

Em várias aplicações práticas é desejável modelar a histerese presente em dispositivos de posicionamento, tais como atuadores piezoelétricos (PZT) e válvulas pneumáticas. $\mathrm{O}$ modelo de BW pode ser adaptado para descrever a histerese presente nesses dispositivos. Para isso, considerase o modelo (1) com $n=1$ (Rakotondrabe, 2011):

$$
\begin{aligned}
\dot{h}(t) & =\alpha_{\mathrm{bw}} \dot{u}(t)-\beta_{\mathrm{bw}}|\dot{u}(t)| h(t)-\gamma_{\mathrm{bw}} \dot{u}(t)|h(t)|, \\
y(t) & =\nu_{y} u(t)-h(t), \quad \nu_{y} \neq 0,
\end{aligned}
$$


em que $u(t)$ é variável de entrada imposta ao dispositivo, $y(t)$ é a posição e $\nu_{y}$ é um parâmetro. Neste artigo, o modelo descrito pelas equações (2) e (3) é referido como o modelo clássico de BW.

Embora essa estrutura seja conhecida, em problemas práticos, os parâmetros devem ser estimados a partir de dados, o que resulta em um problema de otimização não convexo. Em trabalhos recentes, Rakotondrabe (2011) trata o problema de compensação de histerese via inversão do modelo clássico, cujos parâmetros são obtidos usando um método de filtragem não linear. Esse método restringe os parâmetros do modelo clássico a serem estritamente positivos. Tal restrição será removida pelo uso do algoritmo proposto neste artigo quando aplicado a um modelo modificado.

Algoritmos evolutivos destacam-se como ferramentas poderosas e promissoras para resolver problemas de otimização não convexos (Eiben and Smith, 2003). Neste trabalho, investiga-se o problema de estimação de parâmetros para modelos BW usando uma abordagem evolutiva. Tal abordagem é aplicada ao modelo clássico e a um modelo modificado. A modificação proposta consiste na adição de um parâmetro ao modelo clássico e a remoção de restrições sobre o sinal algébrico dos parâmetros estimados. Essa modificação visa tornar o modelo mais maleável. Uma vez estimados os parâmetros, os modelos podem ser usados para mitigar efeitos da histerese. Uma simulação numérica e um sistema físico ilustram a aplicação da abordagem proposta. Os resultados de compensação são comparados entre o modelo clássico, o modelo modificado e o sistema não compensado.

Este trabalho é organizado da seguinte maneira: na Seção 2 são apresentados conceitos sobre algoritmos evolutivos e uma estratégia para compensação de sistemas histeréticos via modelos BW. Na Seção 3 é apresentada a metodologia utilizada para estimar os parâmetros do modelo BW clássico e do modelo modificado. Na Seção 4, tais modelos são identificados e usados para compensar a histerese em um exemplo numérico e um sistema físico. Por fim, a Seção 5 apresenta as conclusões.

\section{FUNDAMENTOS CONCEITUAIS}

Morris (2011) utilizou vários modelos para estudar propriedades da histerese. Um desses foi o modelo BW que apresenta um laço característico independente da frequência da entrada. Essa propriedade é conhecida como histerese independente da taxa, sendo válida para sistemas físicos em que a dinâmica interna é muito mais rápida que as variações da entrada. Assim, transitórios podem ser desconsiderados e o modelo apresenta um continuum de pontos fixos, fato que caracteriza o fenômeno de metaestabilidade.

Pelo fato de existir metaestabilidade, controladores lineares têm seu desempenho deteriorado. Um compensador pode ser projetado para mitigar efeitos histeréticos. Neste artigo, a compensação é realizada via inversão de modelos BW cujos parâmetros são obtidos por meio de um algoritmo evolutivo.

\subsection{Algoritmos Evolutivos}

Em algoritmos evolutivos, o cromossomo é um vetor que contém a representação codificada de um indivíduo, en- quanto o gene é um elemento com uma posição determinada em um cromossomo (Eiben and Smith, 2003). Em problemas de estimação de parâmetros de modelos SISO, tipicamente é utilizada a codificação real. Nessa codificação, cada gene possui um valor real que representa cada parâmetro do modelo. Assim, para uma população de $N_{\text {pop }}$ indivíduos com $n_{v}$ genes, o $i$-ésimo indivíduo possui cromossomo $\boldsymbol{p}_{i}$ que é um vetor de dimensão $n_{v} \times 1$. Cada cromossomo pode ser avaliado via uma função de adaptação que permite comparar esses indivíduos entre si (Fleming and Purshouse, 2002). Os dados $u(k)$ e $y(k)$, $k \in\{1,2, \ldots, N\}$ são conhecidos e são obtidos amostrandose regularmente $u(t)$ e $y(t)$ que são, respectivamente, a entrada e saída do sistema não compensado.

Alguma medida de desempenho é então utilizada para comparar modelos, codificados como cromossomos no algoritmo. Neste trabalho foi utilizado o MAE (do inglês, Mean Absolute Error):

$$
\operatorname{MAE}_{i}=\frac{1}{N} \sum_{k=1}^{N}\left|\hat{y}_{i}(k)-y(k)\right|
$$

em que $\hat{y}_{i}$ é a saída do $i$-ésimo modelo. O objetivo é encontrar o modelo com menor MAE, $\boldsymbol{p}_{*}$.

No transcorrer de um algoritmo evolutivo existem algumas operações típicas como: seleção para cruzamento, cruzamento, mutação e seleção dos sobreviventes. A cada vez que uma sequência dessas operações é concluída, finaliza-se uma geração, sendo o número de gerações $N_{\text {ger geralmente }}$ utilizado como critério de parada. Na seleção para cruzamento, um método típico é o torneio: dois indivíduos são escolhidos aleatoriamente e o mais adaptado é selecionado para o cruzamento (Eiben and Smith, 2003). O número de pais selecionados é determinado pela probabilidade de cruzamento, $P_{\mathrm{c}}$. Com dois desses pais selecionados, o cruzamento aritmético consiste em uma combinação convexa dos genes desses pais. O número de mutações é definido pela probabilidade de mutação $P_{\mathrm{m}}$ e essa operação gera um novo indivíduo a partir de um outro aleatoriamente escolhido. Como indivíduo possui $n_{v}$ genes, o $p$-ésimo gene, $p \in\left\{1,2, \ldots, n_{v}\right\}$ é escolhido via amostragem de uma distribuição normal centrada em zero e de desvio padrão $\sigma_{p}$, em que $\sigma_{p}$ é elemento do vetor $\boldsymbol{\sigma}=\left[\sigma_{1}, \ldots, \sigma_{n_{v}}\right]$.

Na seleção de sobreviventes, um subconjunto de indivíduos é preservado. Para isso, utiliza-se de algum critério de elitismo, de forma a se reduzir as chances de uma busca meramente aleatória. Em problemas não convexos, o elitismo exagerado pode gerar convergência prematura, isto é: a população fica presa em torno de um mínimo local.

A preservação de nichos é uma estratégia que visa promover uma busca global para evitar o problema de convergência prematura. Tal abordagem consiste na divisão dos indivíduos em nichos onde são selecionados os sobreviventes. Ademais, essa estratégia pode ser combinada com uma busca local após um número determinado de gerações.

\subsection{Obtenção do Modelo Inverso $\mathcal{M}_{\mathrm{bw}}^{-1}$}

Seja o modelo $\mathcal{M}_{\text {bw }}$ do sistema $\mathcal{S}$, constituído por (2) e (3). Por meio desse modelo, pode-se obter um compensador via inversão, $\mathcal{M}_{\mathrm{bw}}^{-1}$, conforme Figura 1. Assim, é possível 
determinar a entrada de compensação $u_{\mathrm{c}}(t)$ a ser aplicada ao sistema $\mathcal{S}$. Essa entrada visa aproximar a saída do sistema compensado, $y_{c}(t)$, da referência, $y_{\mathrm{r}}(t)$.

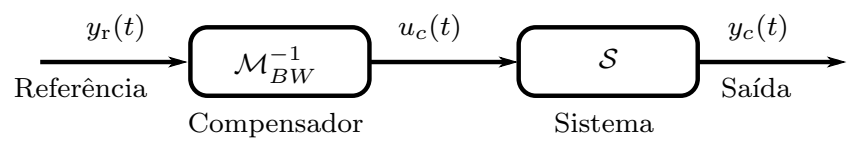

Figura 1. Diagrama de blocos de compensação.

A equação (3) pode ser reescrita para o sistema compensado como: $y_{\mathrm{c}}(t)=\nu_{y} u_{\mathrm{c}}(t)-h(t)$. Como o objetivo é que $y_{\mathrm{c}}(t)$ siga a referência $y_{\mathrm{r}}(t)$, fazemos essa substituição para obter (Rakotondrabe, 2011):

$$
u_{\mathrm{c}}=\frac{y_{\mathrm{r}}+h}{\nu_{y}}
$$

em que $\nu_{y}$ é estritamente positivo e $h$ é obtido de (2). A equação $(5)$ é calculada a cada iteração para gerar a ação de controle $u_{\mathrm{c}}$.

\section{METODOLOGIA}

A equação de saída (3) no presente trabalho foi alterada para

$$
\hat{y}(t)=\hat{\nu}_{y} u(t)+\hat{\mu}_{y} \hat{h}(t), \quad \hat{\nu}_{y} \neq 0,
$$

que apresenta um parâmetro adicional $\hat{\mu}_{y}$. O símbolo "chapéu" foi utilizado em (6) para reforçar o fato de que são os valores de parâmetros ou da saída do modelo estimados e calculados conforme este trabalho. A inserção do parâmetro $\hat{\mu}_{y}$ visa tornar o modelo mais maleável a partir da ponderação da variável histerética e, como será visto na Seção 4, o impacto foi significativo nos resultados obtidos para dois exemplos que foram investigados.

No contexto de identificação de sistemas, possuir um conjunto de dados que excursione vários pontos de operação e excite o sistema em frequências de interesse é fundamental para obtenção de modelos representativos. A Subseção 3.1 apresenta uma abordagem para obter sinais de excitação que sejam coerentes com essas características. Na Subseção 3.2, apresenta-se a estratégia evolutiva utilizada para estimar todos os parâmetros do modelo modificado composto por (2) e (6). O método proposto em (Rakotondrabe, 2011), levemente alterado na Subseção 3.3 foi usado para obter um compensador via inversão do modelo modificado.

\subsection{Sinal de excitação}

De maneira geral em identificação de sistemas, a entrada utilizada tem papel fundamental. No caso de sistemas com histerese a importância de se usar um sinal de excitação adequado é ainda mais crítica. A seguir descreve-se o procedimento seguido no presente artigo. Nota-se que para casos em que os dados não foram gerados seguindo essas recomendações, os modelos identificados não foram capazes de generalizar o comportamento histerético dos sistemas.

O procedimento inicia gerando um sinal puramente aleatório $e(k)=\mathcal{N}(0,1), k=1,2, \ldots N$ em que $\mathcal{N}(0,1)$ indica uma distribuição normal com média 0 e variância 1 . O tempo de amostragem dos dados é igual ao intervalo de integração do modelo BW, ou seja, $T_{\mathrm{s}}=\delta t=0,01 \mathrm{~s}$. Como o fenômeno de histerese é dominante em baixa frequência (Ikhouane and Rodellar, 2007), é necessário concentrar a potência espectral nessa faixa de frequências. Para esse fim foram utilizados filtros passa-baixas do tipo Butterworth de quinta ordem, com frequências de corte $0,01 \mathrm{~Hz}$ e $0,1 \mathrm{~Hz}$. Indicaremos tais filtros por $\mathcal{H}_{0,01}(q)$ e $\mathcal{H}_{0,1}(q)$, respectivamente. As saídas dos filtros são multiplicadas por $\alpha_{0}=\mathrm{G}_{0} / \max [|\mathrm{e}(\mathrm{k})|]$ e $\alpha_{1}=\mathrm{G}_{1} / \max [|\mathrm{e}(\mathrm{k})|]$, respectivamente, de modo que os parâmetros $G_{0}$ e $G_{1}$ são os valores de amplitude máxima para cada uma dessas saídas. Por fim, adiciona-se um valor constante a fim de excursionar o sistema em torno de pontos de operação de interesse. Portanto, os sinais de entrada podem ser gerados como:

$$
u(k)=\left\{\begin{array}{l}
\alpha_{0} \mathcal{H}_{0,01}(q) e(k)+u_{0}, k=1,2, \ldots k_{0} \\
\alpha_{1} \mathcal{H}_{0,1}(q) e(k)+u_{1}, \quad k=k_{0}+1, k_{0}+2, \ldots N .
\end{array}\right.
$$

As frequências de corte dos filtros podem ser adaptadas, se necessário. Tendo investigado diversos outros modelos de histerese, observou-se que os valores aqui utilizados são geralmente adequados.

\subsection{Estimação dos Parâmetros}

Como o modelo modificado é formado por (2) e (6), os parâmetros dessas equações foram estimados a partir de dados amostrados $u(k), y(k)$ e de uma codificação com $n_{v}=5$. O cromossomo do $i$-ésimo indivíduo/modelo é dado por:

$$
\boldsymbol{p}_{i}=\left[\begin{array}{lllll}
\alpha_{\mathrm{bw}}^{i} & \beta_{\mathrm{bw}}^{i} & \gamma_{\mathrm{bw}}^{i} & \hat{\nu}_{y}^{i} & \hat{\mu}_{y}^{i}
\end{array}\right]^{T} .
$$

O espaço de busca é limitado por valores mínimos e máximos para cada gene, ou seja: $\boldsymbol{\ell}_{\min } \leq \boldsymbol{p}_{i} \leq \boldsymbol{\ell}_{\max }$. Além disso, uma restrição adicional deve ser imposta a $\hat{\nu}_{y}^{i}$, devido à necessidade de que esse parâmetro não seja nulo para se realizar a inversão. Dessa forma, visando satisfazer essa condição e evitar possíveis problemas numéricos, impõe-se a restrição $\left|\hat{\nu}_{y}^{i}\right| \geq 10^{-5}$.

Define-se também o número de nichos $N_{\text {nich }}$, sendo que estes dividem o espaço de busca em paralelepípedos de $n_{v}$ dimensões. Cada nicho $j$ tem um centro $\boldsymbol{c}_{j}$ e cada indivíduo $\boldsymbol{p}_{i}$ é rotulado a um único nicho $j$. Essa rotulação é definida pela minimização da norma euclidiana em relação aos centros: $\min _{j}\left\|\boldsymbol{c}_{j}-\boldsymbol{p}_{i}\right\|_{2}$.

Gera-se a população inicial de cada nicho que compõe a população de $N_{\text {pop }}$ indivíduos na geração inicial. Ao longo da primeira metade das gerações, o algoritmo faz uma busca global, mantendo a estratégia de nichos. Definese $\boldsymbol{\sigma}, P_{\mathrm{c}}$ e $P_{\mathrm{m}}$, de modo que $P_{\mathrm{c}}<P_{\mathrm{m}}$ e $P_{\mathrm{m}}>50 \%$. Essas restrições têm por objetivo priorizar a mutação que é mais efetiva na busca global. As operações de seleção via torneio, cruzamento aritmético e mutação são realizadas conforme apresentado na Subseção 2.1. Todos os novos indivíduos são avaliados pelo índice (4) e aqueles provenientes da mutação são novamente rotulados. Ao fim de cada geração, o indivíduo mais adaptado de cada nicho é preservado. Os outros indivíduos sobreviventes são escolhidos aleatoriamente de forma a se manter o tamanho dos nichos. Esse processo se repete até que se alcance a segunda metade de gerações. 
A partir de então, inicia-se uma busca local. Para isso, apenas o nicho em que se localiza o indivíduo mais adaptado é completamente preservado e, para os outros nichos, apenas o indivíduo mais adaptado sobrevive. Fazemos a inversão de prioridade: $P_{\mathrm{c}}>P_{\mathrm{m}}$ e $P_{\mathrm{c}}>50 \%$, de forma a priorizar o cruzamento, mais efetivo na busca local. Para completar a população, são gerados novos indivíduos via mutação dos sobreviventes. Nessa busca, a rotulação por nichos é desconsiderada e as operações de cruzamento e mutação são feitas exatamente como mencionado, mas considerando toda a população. A preservação torna-se mais seletiva: metade dos indivíduos mais adaptados sobrevive e a outra metade é escolhida aleatoriamente sobre os restantes. O algoritmo é finalizado quando o número de gerações ultrapassa $N_{\text {ger }}$.

\subsection{Compensação}

A compensação aqui utilizada é análoga a (5), mas com o parâmetro adicional $\hat{\mu}_{y}$ que foi introduzido na equação (6) e é uma das modificações propostas neste trabalho, ou seja,

$$
\hat{u}_{\mathrm{c}}=\frac{y_{r}-\hat{\mu}_{y} \hat{h}}{\hat{\nu}_{y}}, \hat{\nu}_{y} \neq 0 .
$$

Portanto, tendo estimado os parâmetros como descrito na Subseção 3.2, a cada iteração utiliza-se o modelo (2) para obter $\hat{h}$ por integração numérica. O símbolo "chapéu" é novamente utilizado para reforçar o fato que as variáveis e os parâmetros de (9) são estimadas e calculados conforme este trabalho. Assim, determina-se a ação de controle $\hat{u}_{\mathrm{c}}$ a ser aplicada, uma vez que todas as variáveis do lado direito de (9) são conhecidas. Note também que a condição $\hat{\nu}_{y} \neq 0$ será satisfeita devido às restrições impostas sobre o valor desse parâmetro na etapa de estimação.

\section{RESULTADOS}

Nesta seção, resultados são apresentados para demonstrar a eficiência da abordagem de estimação proposta. Nessa abordagem, pretende-se estimar os parâmetros do modelo modificado e do clássico presente em Rakotondrabe (2011) para dois exemplos: um numérico e outro experimental.

Para o primeiro exemplo a seguir os dados de estimação e de validação são realizações diferentes, mas ambos foram gerados como apresentado na Subseção 3.1. No segundo exemplo, em função de limitações físicas, apenas um filtro foi utilizado, como será detalhado na Subseção 4.2. Outros conjuntos de validação são gerados a partir de entradas senoidais de diversas frequência e amplitudes.

Como os modelos incluem uma equação diferencial, (2), utiliza-se o método Runge-Kutta de $4^{\mathrm{a}}$ ordem com passo de integração $\delta t=0,01$ s. O índice MAPE (do inglês, Mean Absolute Percentage Error) é o critério para quantificação dos resultados em relação à faixa de excursão:

$$
\mathrm{MAPE}=\frac{\mathrm{MAE}}{|\max [y(k)]-\min [y(k)]|} .
$$

Os meta parâmetros do algoritmo evolutivo, comuns à identificação em ambos os exemplos, são: $N_{\text {ger }}=100$, $P_{\mathrm{c}}^{\mathrm{g}}=50 \%, P_{\mathrm{c}}^{\mathrm{l}}=100 \%, P_{\mathrm{m}}^{\mathrm{g}}=90 \%, P_{\mathrm{m}}^{\mathrm{l}}=10 \%, \sigma^{\mathrm{g}}=$ $20 \% \times\left(\boldsymbol{\ell}_{\max }-\ell_{\min }\right)$ e $\boldsymbol{\sigma}^{\mathrm{l}}=10 \% \times \boldsymbol{\sigma}^{\mathrm{g}}$, em que l e g denotam valores para à busca local e à global, respectivamente. $\mathrm{O}$ número de nichos usado foi $N_{\text {nich }}=2^{n_{v}}$ com 10 indivíduos em cada nicho, $N_{\text {pop }}=10 N_{\text {nich }}$. Os seguintes limites foram definidos: $\boldsymbol{\ell}_{\max }=\left[\begin{array}{lllll}10 & 10 & 10 & 10 & 10\end{array}\right]^{T}$ e $\boldsymbol{\ell}_{\min }=-\boldsymbol{\ell}_{\max }$.

Para estimar os parâmetros do modelo clássico (2) e (3) pela estratégia da Subseção 3.2, algumas alterações são realizadas. Devido a escolha de $\mu_{y}=-1$, uma modificação do $i$-ésimo cromossomo (8) é necessária, tal que:

$$
\boldsymbol{q}_{i}=\left[\begin{array}{llll}
\alpha_{\mathrm{bw}}^{i} & \beta_{\mathrm{bw}}^{i} & \gamma_{\mathrm{bw}}^{i} & \nu_{y}^{i}
\end{array}\right]^{T},
$$

sendo o espaço de busca limitado a valores de parâmetros que sejam estritamente positivos (Rakotondrabe, 2011): $\ell_{\max }=\left[\begin{array}{llll}10 & 10 & 10 & 10\end{array}\right]^{T}$ e $\boldsymbol{\ell}_{\min }=10^{-5} \times\left[\begin{array}{llll}1 & 1 & 1 & 1\end{array}\right]^{T}$.

Ao final das gerações, objetiva-se encontrar os cromossomos ótimos $\boldsymbol{p}_{*}$ e $\boldsymbol{q}_{*}$ que correspondem aos parâmetros do modelo modificado e do clássico, respectivamente. É importante salientar que o modelo modificado apresenta duas modificações importantes em relação ao modelo clássico: a primeira se deve à presença do parâmetro $\hat{\mu}_{y}$ e a segunda se refere à possibilidade dos parâmetros possuírem valores negativos. Nos exemplos estudados, uma dessas características ou ambas permitiram que os modelos modificados apresentassem resultados superiores nos processos de identificação e compensação. Nas subseções seguintes os modelos estimados serão comparados em termos de seu comportamento dinâmico. Por fim, esses modelos são utilizados no projeto de compensação, de modo a mitigar os efeitos da histerese presente em ambos sistemas.

\subsection{Um Exemplo Numérico - Prandtl-Ishlinskii}

Um modelo de Prandtl-Ishlinskii (PI) é apresentado em Edardar et al. (2014) para representar um atuador PZT de nanoposicionamento. Tal modelo é aqui utilizado como sistema $\mathcal{S}$, sendo composto por 5 operadores com limiares expressos em $\boldsymbol{r}=\left[\begin{array}{lllll}0 & 0,63 & 1,27 & 2,54 & 4,45\end{array}\right]^{T}$ e pesos em $\boldsymbol{w}=\left[\begin{array}{lllll}5,88 & 1,58 & 0,47 & 0,98 & 0,4\end{array}\right]^{T}$.

Foi gerado um sinal de entrada de estimação conforme (7) $\operatorname{com} N=6000, u_{0}=5 \mathrm{~V}, \mathrm{G}_{0}=4 \mathrm{~V}, k_{0}=4800, u_{1}=10 \mathrm{~V}$ e $\mathrm{G}_{1}=3 \mathrm{~V}$. Um segundo sinal de entrada foi gerado de forma similar para a validação dos modelos (Figura 2). Conforme pode ser observado na Figura 2a, os dados de saída se localizam em torno de $50 \mu \mathrm{m}$ e $100 \mu \mathrm{m}$. A escolha de $k_{0}$ foi realizada de tal modo que o número de oscilações em torno de cada ponto de operação fosse próximo.

Após coletar os dados de identificação, aplica-se a estratégia evolutiva para obter os parâmetros do modelo modificado. Os parâmetros são apresentados conforme (8):

$$
\boldsymbol{p}_{*}=\left[\begin{array}{lllll}
0,65 & 0,21 & 0,66 & 9,28 & 3,98
\end{array}\right]^{T} .
$$

$\mathrm{O}$ item $\mathrm{A}_{1}$ da Tabela 1 , mostra o MAPE desse modelo para entradas senoidais.

Para comparar as modificações, obtemos os parâmetros do modelo clássico (2) e (3), dados por:

$$
\boldsymbol{q}_{*}=\left[\begin{array}{llll}
1,97 & 0,24 & 0,022 & 9,29
\end{array}\right]^{T},
$$

de modo que $\boldsymbol{q}_{*}$ está codificado como em (11). Os resultados de MAPE para o modelo clássico são indicados no item $\mathrm{B}_{1}$ da Tabela 1 . Os resultados do modelo proposto neste artigo (ver item $A_{1}$ ) são superiores. $O$ erro percentual 
médio e máximo do modelo clássico em relação ao proposto é de $48 \%$ e $135 \%$, respectivamente.
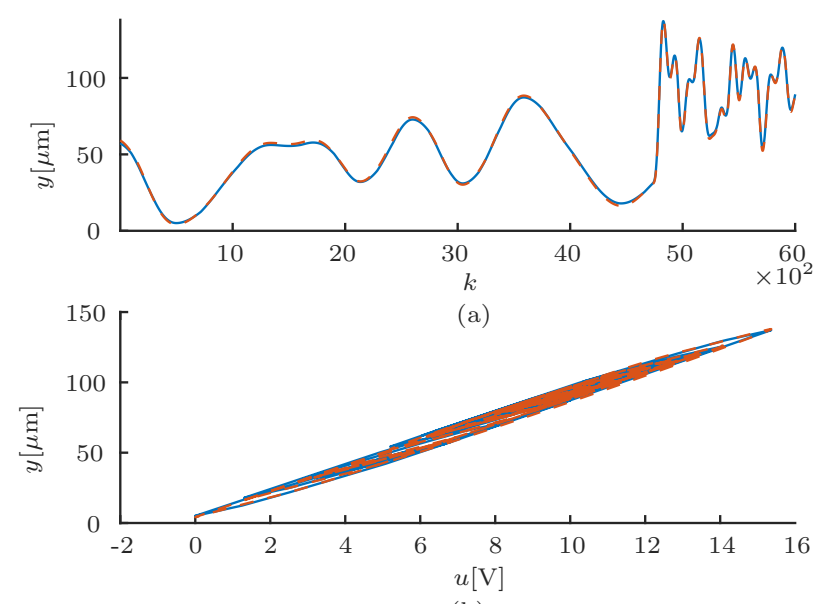

(b)

Figura 2. Simulação livre em dados de validação. A linha contínua em azul (-) apresenta os dados do sistema PI, enquanto a linha tracejada em vermelho (- -) apresenta os dados do modelo com parâmetros (12). (a) evolução temporal e (b) o plano entrada $\times$ saída.

Tabela 1. MAPE expresso em $\%$ dos modelos $A_{1}$ e $B_{1}$ para entradas senoidais. $O$ modelo $A_{1}$ é dado por (2) e (6) com parâmetros (12). O modelo $\mathrm{B}_{1}$ por (2) e (3) e parâmetros (13).

\begin{tabular}{c|c|cccc} 
& & \multicolumn{4}{|c}{ Amplitude [V] } \\
\hline \multirow{3}{*}{$\mathrm{A}_{1}:(12)$} & $\mathrm{f}[\mathrm{Hz}]$ & 1 & 5 & 10 & 50 \\
\hline & 0,01 & 3,54 & 0,47 & 0,39 & 0,13 \\
& 0,1 & 3,50 & 0,52 & 0,47 & 0,27 \\
& 0,5 & 3,41 & 0,93 & 1,03 & 1,01 \\
$\mathrm{~B}_{1}:(13)$ & 1 & 3,46 & 1,59 & 1,82 & 1,94 \\
\hline & 0,01 & 5,11 & 0,62 & 0,70 & 0,21 \\
& 0,1 & 5,08 & 0,61 & 0,76 & 0,36 \\
& 0,5 & 5,02 & 0,99 & 1,26 & 1,09 \\
& 1 & 5,09 & 1,70 & 2,06 & 2,04
\end{tabular}

Para a compensação via modelo inverso obtido dos parâmetros (12), apresentamos a comparação da saída do sistema compensado com a referência senoidal de $50 \mu \mathrm{m}$ e $0,1 \mathrm{~Hz}$ na Figura 3. Utilizando o compensador, a relação entre a saída e a referência são praticamente lineares (Figura $3 \mathrm{~b}$ ), fato que não ocorre na ausência de compensação.

$\mathrm{O}$ item $\mathrm{A}_{2}$ da Tabela 2 apresenta os valores de MAPE do sistema compensado via modelo modificado para referências senoidais de diversas frequências e amplitudes. Por outro lado, o item $\mathrm{B}_{2}$ apresenta os valores de MAPE da compensação para o modelo clássico. Por meio da Tabela 2, fica claro que o compensador $\mathrm{B}_{2}$ é inferior ao compensador $\mathrm{A}_{2}$. $\mathrm{O}$ erro médio do modelo clássico em relação ao modificado é da ordem de 5 vezes, enquanto o erro máximo da ordem de 30 vezes. O desempenho superior da compensação realizada com o modelo $\mathrm{A}_{1}$ (Tabela 1 ) indica que, como esperado, o procedimento é sensível à qualidade do modelo. Embora o algoritmo não tenha selecionado parâmetros negativos para o modelo modificado, a inserção de $\hat{\nu}_{y}$ torna o modelo modificado mais representativo. Esse fato é corroborado pelos resultados de validação do modelo e compensação (ver Tabelas 1 e 2). Observe também que foi obtido $\hat{\nu}_{y}=9,28 \gg 0$, viabilizando a inversão do modelo.

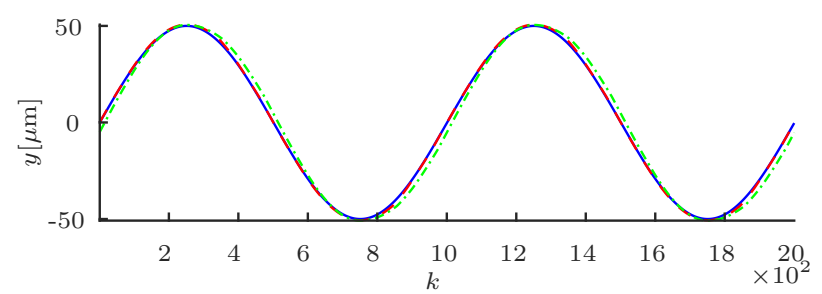

(a)

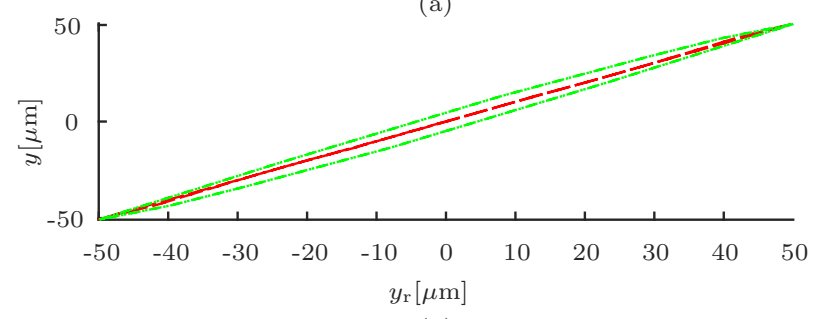

(b)

Figura 3. Saída do sistema PI com e sem compensação via modelo com parâmetros (12). A referência é apresentada pela linha contínua azul (-), a saída do sistema compensado pela linha tracejada em vermelho (- -) e os traços e pontos em verde (---) referem-se a saída sem compensação. (a) evolução temporal e (b) laços entre a referência e essas saídas.

Tabela 2. MAPE expresso em \% para as saídas dos sistemas compensados $\mathrm{A}_{2}$ e $\mathrm{B}_{2}$ quando as referências são senoidais. $\mathrm{O}$ modelo $\mathrm{A}_{2}$ é dado por (5) com parâmetros (13), enquanto o modelo $\mathrm{B}_{2}$ por (9) e parâmetros (12).

\begin{tabular}{c|c|cccc} 
& & \multicolumn{4}{|c}{ Amplitude [V] } \\
& $\mathrm{f}[\mathrm{Hz}]$ & 20 & 50 & 100 & 400 \\
\hline & 0,01 & 0,94 & 0,46 & 0,36 & 0,16 \\
$\mathrm{~A}_{2}:(12)$ & 0,1 & 0,96 & 0,49 & 0,38 & 0,16 \\
& 0,5 & 1,14 & 0,68 & 0,49 & 0,19 \\
& 1 & 1,48 & 0,92 & 0,63 & 0,23 \\
\hline & 0,01 & 5,11 & 0,61 & 0,69 & 0,21 \\
$\mathrm{~B}_{2}:(13)$ & 0,1 & 5,08 & 0,61 & 0,75 & 0,35 \\
& 0,5 & 5,03 & 0,98 & 1,26 & 1,09 \\
& 1 & 5,09 & 1,69 & 2,04 & 2,03
\end{tabular}

\subsection{Aplicação a uma Válvula Pneumática}

Este exemplo considera uma válvula pneumática, atuador de uma planta piloto de nível. Tal válvula é alimentada por tensão elétrica e opera ao deslocar um êmbolo regulador da passagem de fluxo. Assim, a entrada do sistema é essa tensão, enquanto a saída é a posição do êmbolo. A Figura 4 apresenta uma fotografia dessa válvula.

Para verificar a presença de histerese nessa válvula, estudos anteriores foram desenvolvidos por Ribeiro (2017) e Nunes (2019). Recentemente, Aguirre (2019) mostrou como procedimentos de identificação caixa cinza podem ser utilizados para obter modelos polinomiais NARX (do inglês, Nonlinear AutoRegressive with eXogenous inputs) capazes de reproduzir a histerese presente nessa válvula.

Além do mais, é possível utilizar modelos independentes da taxa para representar a histerese em válvulas desse tipo. Um exemplo é o modelo de Duhem (Ikhouane, 2018) que já foi utilizado para esse fim conforme Zhou and Wen (2008). Nesse sentido, justifica-se utilizar modelos fenomenológicos independentes da taxa como BW para modelagem desse 
fenômeno. A seguir, utiliza-se o modelo clássico e o modelo modificado para a modelagem e compensação de histerese nessa válvula.

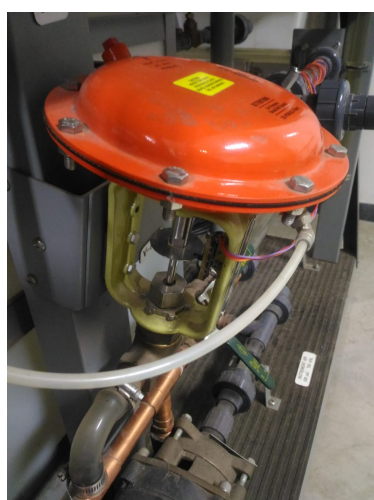

Figura 4. Fotografia da válvula utilizada neste trabalho.

Nos resultados desta subseção, tanto a entrada quanto a saída são apresentadas variando dentre os limites de $0 \%$ a $100 \%$. A entrada de identificação é gerada a partir da aplicação dos parâmetros $N=6000, u_{0}=50 \%, \mathrm{G}_{0}=45 \%$, $k_{0}=6000$ à equação (7), de modo que a filtragem só ocorre para a frequência de $0,01 \mathrm{~Hz}$. Ao aplicar a estratégia evolutiva de maneira análoga à subseção anterior, obtemos:

$$
\begin{aligned}
\boldsymbol{p}_{*} & =\left[\begin{array}{lllll}
-3,08 & -3,06 & -1,83 & 0,04 & 0,16
\end{array}\right]^{T}, \\
\boldsymbol{q}_{*} & =\left[\begin{array}{llll}
0,69 & 1,92 & 0,48 & 1,01
\end{array}\right]^{T},
\end{aligned}
$$

sendo $\boldsymbol{p}_{*}$, os parâmetros dados na codificação (8) e $\boldsymbol{q}_{*}$ na codificação (11).

Na Figura 5, tem-se a validação para o modelo modificado (14) quando aplicada uma senóide de frequência $0,01 \mathrm{~Hz}$, média $50 \%$ e amplitude $28 \%$. Observe que mesmo com a presença de ruído de medição, tal modelo se ajusta aos dados, sendo possível a representação do laço de histerese. Contudo, o modelo perde aderência nas regiões de máximos e mínimos da saída.
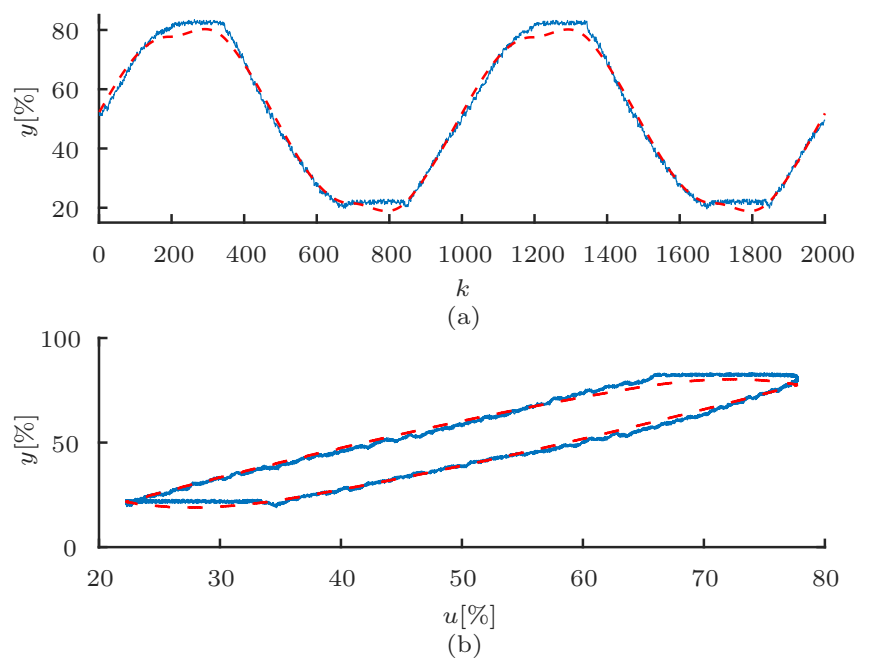

Figura 5. Simulação livre em dados de validação. A linha contínua em azul (-) apresenta os dados medidos da válvula, enquanto a linha tracejada em vermelho (- -) mostra os dados do modelo com parâmetros (14). (a) evolução temporal e (b) o plano entrada $\times$ saída.

A compensação é realizada para referências senoidais de média $50 \%$, frequência $0,01 \mathrm{~Hz}$ e diversas amplitudes. A
Figura 6 apresenta $\hat{u}_{\mathrm{c}}(9)$ e $u_{\mathrm{c}}(5)$ para uma referência $y_{\mathrm{r}}$ senoidal de amplitude 30\%. Essas entradas são calculadas via inversão dos modelos dados pelos parâmetros (14) e (15), respectivamente.

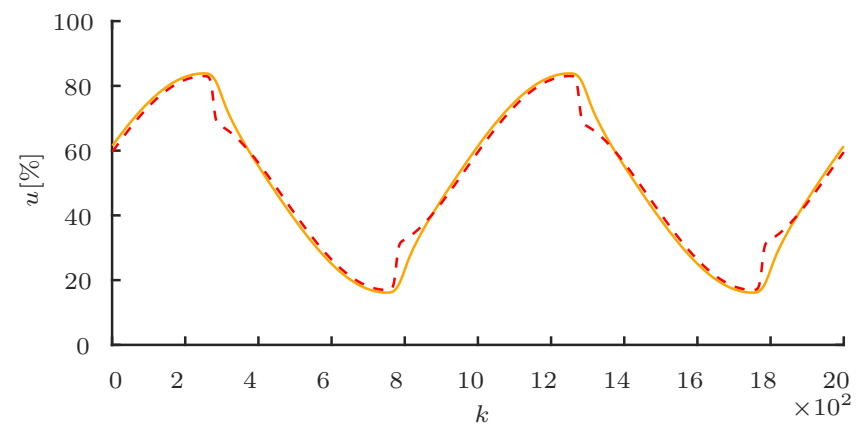

Figura 6. Entradas de compensação calculadas para válvula quando o modelo inverso é alimentado por uma referência senoidal de média $50 \%$, frequência $0,01 \mathrm{~Hz}$ e amplitude $30 \%$. A linha tracejada em vermelho (- -) apresenta a entrada obtida dos parâmetros (14), enquanto a linha contínua em laranja (-) se refere aos parâmetros (15).

As Figuras 7 e 8 apresentam o desempenho dos sistemas compensados para uma referência de amplitude $30 \%$. A compensação é deteriorada na região de extremos da senóide devido ao fato de o modelo ser menos acurado nessa região, conforme pode ser visto na Figura 5. Observe que a válvula compensada têm desempenho superior ao caso não compensado, independente da compensação usada. Contudo, ao se utilizar o modelo modificado de parâmetros (14), tem-se uma compensação mais efetiva, fato que se comprova na comparação dos laços presentes nas Figuras 7 e 8 .

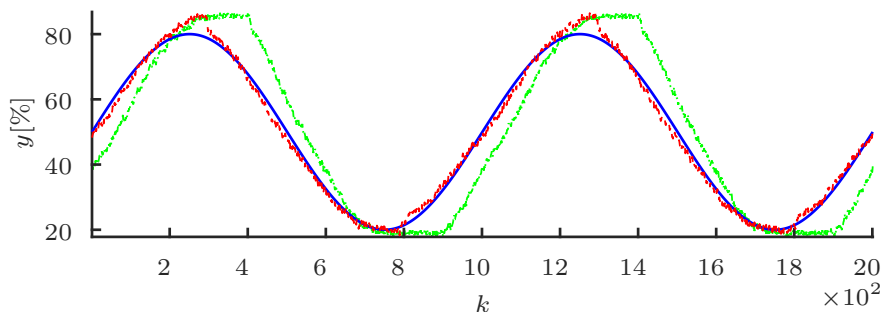

(a)

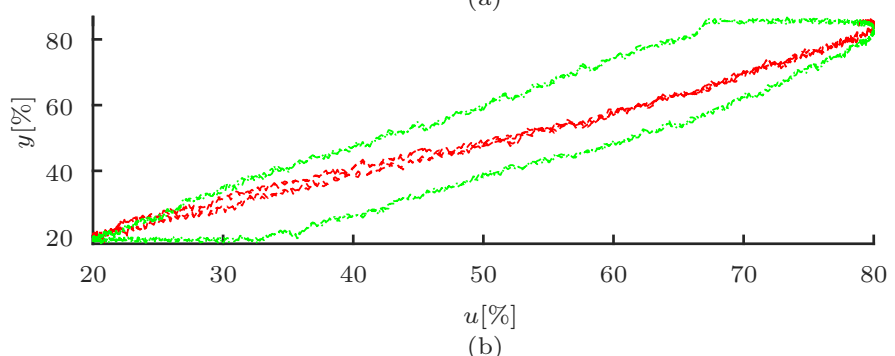

Figura 7. Desempenho da válvula sem compensação traços e pontos em verde (---) - e compensada via modelo com parâmetros (14) - linha tracejada em vermelho (- -). A referência é apresentada pela linha contínua azul (-). (a) evolução temporal; (b), os laços entre a referência e essas saídas.

Os resultados para testes realizados com referências de outras amplitudes estão na Tabela 3. Esses resultados também comprovam que o modelo modificado apresentou melhores resultados de compensação em todos os cenários 
avaliados. O modelo clássico apresentou um erro médio de $47 \%$ e máximo de $62 \%$ em relação ao modelo modificado. A perda de qualidade na compensação a partir do modelo clássico está ligada a menor representatividade desse modelo conforme atesta a Tabela 3 .
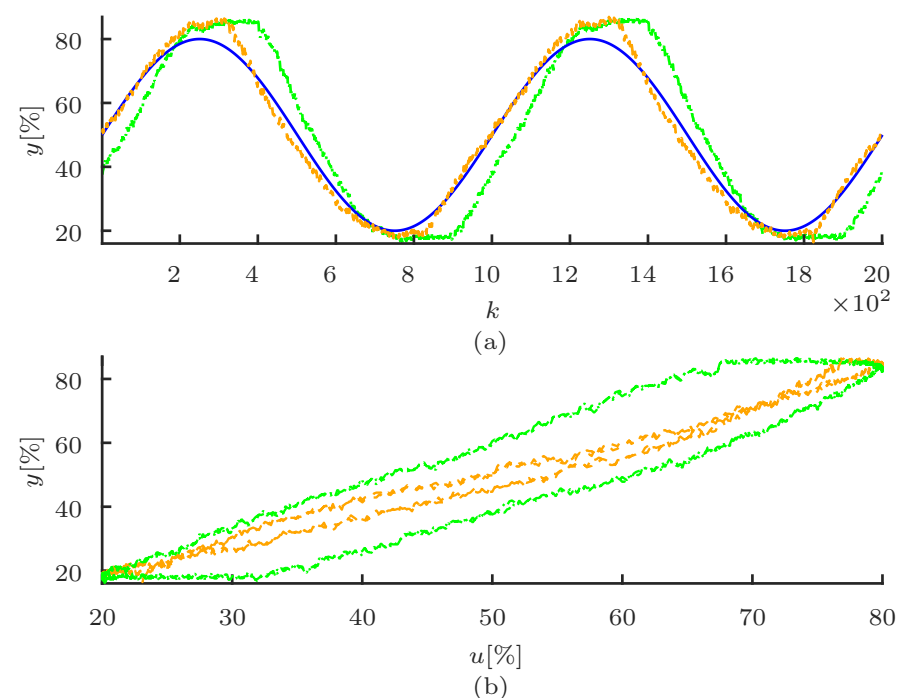

Figura 8. Desempenho da válvula sem compensação traços e pontos em verde (---) - e compensada via modelo com parâmetros (15) - linha tracejada em laranja (- -). A referência é apresentada pela linha contínua azul (-). (a) evolução temporal; (b), os laços entre a referência e essas saídas.

Tabela 3. MAPE expresso em \% para as saídas dos sistemas compensados $\mathrm{A}_{3}$ e $\mathrm{B}_{3}$ quando as referências são senoidais de $0,01 \mathrm{~Hz}$. O modelo modificado $\mathrm{A}_{3}$ tem parâmetros (14). O modelo clássico $\mathrm{B}_{3}$ tem parâmetros (15).

\begin{tabular}{c|ccc}
\multirow{2}{*}{ Modelos } & \multicolumn{3}{|c}{ Amplitude [\%] } \\
& 20 & 30 & 40 \\
\hline $\mathrm{A}_{3}:(14)$ & 3,20 & 2,39 & 2,35 \\
\hline $\mathrm{B}_{3}:(15)$ & 4,83 & 3,85 & 3,00
\end{tabular}

\section{CONCLUSÕES}

Este trabalho apresentou uma abordagem evolutiva para obtenção de parâmetros do modelo Bouc-Wen que é utilizado na compensação de sistemas histeréticos. Como essa abordagem permite a inserção de parâmetros com facilidade, uma parâmetro é adicionado ao modelo clássico apresentado em Rakotondrabe (2011), gerando um modelo modificado. Além do parâmetro adicional, o modelo modificado permite que todos os parâmetros apresentem valores negativos, o que não ocorre com o modelo clássico. Ambos modelos foram usados para realizar a compensação de histerese em dois casos: uma simulação numérica do modelo de Prandtl-Ishlinskii dado em (Edardar et al., 2014) e um sistema físico, válvula pneumática de uma planta piloto de nível. Em todos os casos avaliados, o modelo clássico apresentou desempenho inferior em relação ao modelo modificado, embora também tenha sido útil para compensar parte dos efeitos não lineares.

Apesar de essa abordagem ter sido capaz de resolver os problemas estudados, algoritmos evolutivos são computacionalmente custosos. Propõe-se para trabalhos futuros estudar o problema de compensação usando modelos que requeiram menor esforço computacional, como os polinômios NARX.

\section{REFERÊENIAS}

Aguirre, L.A. (2019). A bird's eye view of nonlinear system identification. ArXiv:1907.06803v2.

Bouc, R. (1967). Forced vibration of mechanical systems with hysteresis. In Proceedings of the Fourth Conference on Nonlinear Oscillations, 32-39. Prague, Czechoslovakia.

Cross, R., Grinfield, M., and Lamba, H. (2009). Hysteresis and economics. IEEE Control Systems, 29(1), 30-43.

Edardar, M., Tan, X., and Khalil, H.K. (2014). Tracking error analysis for feedback systems with hysteresis inversion and fast linear dynamics. Journal of Dynamic Systems, Measurement and Control, 136(4), 041010.

Eiben, A. and Smith, J. (2003). Introduction to Evolutionary Computing. Springer.

Fleming, P.J. and Purshouse, R.C. (2002). Evolutionary algorithms in control systems engineering: a survey. Control Engineering Practice, 10, 1223-1241.

Ikhouane, F. and Rodellar, J. (2007). Systems with Hysteresis: Analysis, Identification and Control Using the Bouc-Wen Model. Wiley.

Ikhouane, F. (2018). A survey of the hysteretic duhem model. Archives of Computational Methods in Engineering, 25(4), 965-1002.

Morris, K.A. (2011). What is hysteresis? Applied Mechanics Reviews, 64, 050801.

Nunes, G.M. (2019). Modelagem de histerese de atuador e análise de desempenho de controladores digitais para controle de planta piloto (no prelo). Monografia de Graduação em Engenharia de Controle e Automação, Escola de Engenharia, Universidade Federal de Minas Gerais, Belo Horizonte.

Rakotondrabe, M. (2011). Bouc-wen modeling and inverse multiplicative structure to compensate hysteresis nonlinearity in piezoelectric actuators. IEEE Transactions on Automation, Science and Engineering, 8(2), 1-28.

Ribeiro, M.A.G. (2017). Modelagem e controle digital em malha fechada de uma planta de nível. Monografia de Graduação em Engenharia Elétrica, Escola de Engenharia, Universidade Federal de Minas Gerais, Belo Horizonte.

Sha, W. et al. (2003). Hysteresis drives cell-cycle transitions in xenopus laevis egg extracts. PNAS, 100(3), 975-980.

Swevers, J. et al. (2000). An integrated friction model structure with improved presliding behavior for accurate friction compensation. IEEE Transactions on Automation, Science and Engineering, 45(4), 675-686.

Visone, C. (2008). Hysteresis modelling and compensation for smart sensors and actuators. Journal of Physics: Conference Series, 138, 1-28.

Wen, Y.K. (1976). Method for random vibration of hysteresis systems. Journal of the Engeneering Mechanics Division, 102(2), 249-263.

Zhou, J. and Wen, C. (2008). Adaptive backstepping control of uncertain systems: nonsmooth nonlinearities, interactions or time-variations. Springer. 